Nat Protoc. ; 6(10): 1632-1641. doi:10.1038/nprot.2011.397.

\title{
Using the Drosophila melanogaster D17-c3 cell culture system to study cell motility
}

\author{
Joshua D Currie ${ }^{1}$ and Stephen L Rogers ${ }^{1,2,3}$ \\ ${ }^{1}$ Department of Biology, The University of North Carolina at Chapel Hill, Chapel Hill, North \\ Carolina, USA \\ ${ }^{2}$ Carolina Center for Genome Sciences, The University of North Carolina at Chapel Hill, Chapel \\ Hill, North Carolina, USA \\ ${ }^{3}$ Lineberger Comprehensive Cancer Center, The University of North Carolina at Chapel Hill, \\ Chapel Hill, North Carolina, USA
}

\begin{abstract}
Cultured Drosophila melanogaster S2 and S2R + cell lines have become important tools for uncovering fundamental aspects of cell biology as well as for gene discovery. Despite their utility, these cell lines are nonmotile and cannot build polarized structures or cell-cell contacts. Here we outline a previously isolated, but uncharacterized, Drosophila cell line named Dm-D17-c3 (or D17). These cells spread and migrate in culture, form cell-cell junctions and are susceptible to RNA interference (RNAi). Using this protocol, we describe how investigators, upon receiving cells from the Bloomington stock center, can culture cells and prepare the necessary reagents to plate and image migrating D17 cells; they can then be used to examine intracellular dynamics or observe loss-of-function RNAi phenotypes using an in vitroscratch or wound healing assay. From first thawing frozen ampules of D17 cells, investigators can expect to begin assaying RNAi phenotypes in D17 cells within roughly 2-3 weeks.
\end{abstract}

\section{INTRODUCTION}

Cultured Drosophila melanogaster cells have become powerful genetic tools for the study of numerous cell biological processes. Established lines such as S2 and S2R + offer highresolution cytology, simple culture conditions, a fairly homogeneous morphology when seeded on the lectin concanavalin A, and, most notably, a potent susceptibility to RNAi that can be applied on a genomic level ${ }^{1-5}$. These benefits are additionally advantageous because of the succinct nature of the fly genome ${ }^{6}(\sim 14,000$ genes) and the potential to easily move cell culture observations into a model organism ${ }^{7}$. The end result has been a cell culture

(C) 2011 Nature America, Inc. All rights reserved.

Correspondence should be addressed to S.R. (srogers@bio.unc.edu)..

AUTHOR CONTRIBUTIONS J.D.C. performed the protocol under the supervision of S.L.R. J.D.C. and S.L.R. wrote the protocol manuscript.

COMPETING FINANCIAL INTERESTS The authors declare no competing financial interests.

Supplementary information is available via the HTML version of this article. 
system that has been applied to disciplines as diverse as developmental biology ${ }^{8-10}$, microbiology ${ }^{11}$ and high-throughput functional genomics ${ }^{12,13}$.

Although lines such as S2 and S2R + have advanced our understanding of the basic mechanisms underlying cell morphology and cytoskeletal organization, their use is somewhat limited in this regard because of their lack of true cell locomotion. Moreover, these cell lines usually exist in culture as single, rounded cells that lack characteristics such as cell-cell junctions, which are necessary structures for organismal development.

The Drosophila Genomics Resource Center (DGRC, http://dgrc.cgb.indiana.edu/) in Bloomington, Indiana, is a repository for dozens of Drosophila cell lines derived from various fly tissues at different developmental stages. Many of these cell lines have remained uncharacterized with respect to their ability to respond to RNAi or their real-time dynamics in culture.

One such line, Dm-D17-c3 (hereafter referred to as D17), was isolated in addition to roughly two dozen other cell lines in the lab of the late Tadashi Miyake at the Mitsubishi-Kasei Institute of Life Sciences in Tokyo. Although only half of these lines were ever published ${ }^{14}$, the entire collection of cell lines isolated from third instar larvae is currently available at the DGRC (http://dgrc.cgb.indiana.edu/cells/store/catalog.html?category=2). The D17 and their sibling lines were cultured from dissected imaginal discs, pools of multipotent cells in the developing Drosophila larvae responsible for creating the vast array of adult tissue needed during metamorphosis.

\section{Characteristics of D17}

When observing D17 cells in culture, the cells show a more heterogeneous set of morphologies than S2 cells, but usually spread and form islands of epithelial-like colonies or migrate nondirectionally as single cells. In confluent culture, the cells often form a complete monolayer, but are not classically contact inhibited, resulting in foci of cells that build up above the surface of the cell monolayer. Time-lapse video microscopy of D17 cells revealed a robust motility of approximately $25-30 \mu \mathrm{m} \mathrm{h}^{-1}$ (Fig. 1), similar to the initial speeds of Drosophila hemocytes in vivo as they migrate along the ventral midline ${ }^{15}\left(\sim 24 \mu \mathrm{m} \mathrm{h}^{-1}\right)$. Additionally, when migrating, these cells adopt a morphology reminiscent of mammalian cells migrating in culture; producing a polarized, triangular organization with a large fanlike lamella at the leading edge and trailing retraction fibers in the rear of the cell (Fig. 2 and Supplementary Video 1).

Although the D17 cells were originally believed to be epithelial cells due to the nature of the tissue they were derived from ${ }^{14}$, the cells have properties reminiscent of Drosophila hemocytes, which are migratory Drosophila immune cells. This is evident from their similar morphological properties during migration ${ }^{16}$ as well as the propensity of D17 for phagocytic clearance. Indeed, we often observe D17 cells in culture that exhibit a chemotactic attraction to the debris of their apoptosed neighbors and even undergo whole-cell engulfment (J.D.C. and S.L.R., unpublished observations). D17 cells also mimic hemocytes in their ability to secrete extracellular matrix (ECM), which they require for migration (see Box 1). In addition to this observational evidence for the D17's possible cell lineage, thanks to recent 
genomic information available from the modENCODE project ${ }^{17}$ (http://

www.modencode.org), we can make inferences about the differentiated state of the D17 cell line based on their transcriptional profile. Using these online tools we have found that, similarly to S2 and S2R + cells, D17 cells express elevated message levels of the hemocytespecific transcription factor Serpent ${ }^{18}$ as well as other hemocyte-specific genes, suggesting that these two cell lines may have comparable expression profiles. Recent work from the modENCODE project has further characterized the transcriptional profile of 25 established Drosophila cell lines, including the D17 cell line ${ }^{19}$. Expression profile clustering demonstrated that D17 cells share broad expression similarities to embryonic S2R + cells. However, on the basis of the expression of the homeotic transcription factor, teashirt ${ }^{20}$, in D17 cells, the authors conclude that D17 cells are indeed imaginal haltere disc cells ${ }^{19}$. This conclusion is somewhat confounded by the fact that embryonic S2 and S2R + cells also express relatively high levels of teashirt based on modENCODE expression data and it seems that this fact alone does not explicitly reveal the identity of the D17 cell line. Further work will be necessary to establish the identity of the D17 cell line based on gene expression, cell biological observations and biochemical analysis between various Drosophila cell lines. Hopefully, continued use of the D17 cell line will further open up intriguing questions about the mechanisms of cell identity, cell morphology and cell motility.

\section{Advantages and key applications of D17}

The key component that makes Drosophila cells an advantageous system is their potent susceptibility to RNAi and the relative simplicity of producing and treating cells with double-stranded RNA (dsRNA). Generally, this procedure involves creating dsRNA that is transcribed from a target DNA template with flanking T7 RNA polymerase start sites. Addition of the produced dsRNA to cell growth medium is sufficient to induce cellular uptake and gene depletion ${ }^{3}$ without the use of lipid-based transfections or virus infection. This methodology has been greatly enhanced by commercial and academic resources that provide online tools for designing dsRNA primers (Harvard Drosophila RNAi Screening Center (DRSC), http://www.flyrnai.org), obtaining cDNA templates (Open Biosystems, http://www.openbiosystems.com) and procuring genome-wide as well as custom sublibraries (Harvard DRSC) of RNAi targets.

The utility of the D17 cell line should augment the studies of many in the cell biology and Drosophila communities who wish to study target genes and pathways in the context of biological processes such as cell migration and cell-cell interactions (Fig. 3). In addition, many of the protocols already established for S2 cell culture ${ }^{21}$ can easily be adapted for D17 cell culture. The D17 cell line represents an untapped resource to answer existing questions in various fields as well as provide a model to ask new questions about the genetic basis of cell migration, chemotaxis and cell differentiation.

\section{Overview of the procedure}

We outline how to thaw frozen ampules from the DGRC and establish cultures that can be passaged; prepare D17 growth medium; and isolate a crude ECM preparation from conditioned media that allows users to plate D17 cells on glass surfaces. We will also 
outline how to experimentally assay D17 cells by transient transfection, dsRNA treatment, and examine migration through a classical wound healing assay that can be used to analyze RNAi phenotypes. Using the protocols outlined here, we have previously been able to demonstrate the negative regulation of D17 migration by the microtubule-severing enzyme, $\operatorname{katanin}^{22}$.

\section{Experimental design}

RNAi-D17 cells can be treated in much the same way as common S2 and S2R + lines (Steps 10-14). For any RNAi experiment, it is important that users include important RNAi controls to ensure that RNAi phenotypes are penetrant and effectively affect migration. We recommend using the small GTPase Rho as a positive control for penetrant RNAi phenotypes in S2 cells and D17 cells, as its knockdown can be assayed by western blotting (Fig. 1e) and produces an easily visible multinucleate phenotype (Fig. 1d $)^{21,23}$.

As a positive control for RNAi migration phenotypes, we suggest using the Drosophila Arp2/3 activator SCAR. SCAR exists as a single isoform in Drosophila and markedly inhibits D17 migration (Fig. 1c). RNAi against the small GTPase Rac can also be used to inhibit D17 migration, but investigators should note the compensatory effects of the two Drosophila Rac isoforms and related GTPase $\mathrm{Mtl}^{24,25}$. Control-treated cells can be treated with dsRNA made against the Bluescript plasmid ${ }^{21}$.

The depletion of target proteins can vary between cell lines depending on the differential stability or expression level of the target polypeptide as well as the kinetics of protein dilution through sequential cell divisions, making a direct comparison of RNAi efficacy between S2 cells and D17 cells tenuous at best. Immunoblots of tubulin and Rho from equally loaded lysates reveal that S2 and D17 cells express different levels of both proteins. Seven-day treatment of dsRNA using the protocol outlined here results in a similar degree of knockdown between cell lines (Fig. 1e). We suspect that the kinetics of protein depletion may be slightly slower in D17 cells as a result of their longer doubling time of 48-72 h versus a 24-h doubling time in S2 cells (J.D.C. and S.L.R., unpublished observations). In our hands, potent depletion (greater than $80 \%$ knockdown) in D17 cells can usually be obtained within 7-9 d. We recommend that investigators empirically optimize the length of dsRNA treatment in D17 cells for each RNAi target to achieve the optimal knockdown.

Isolation of the ECM-The methodology described in Box 1 is based on the early purification steps for isolating Drosophila ECM components outlined by John Fessler ${ }^{26}$ of the University of California, Los Angeles. We have not yet identified the specific ECM components or factors that support D17 growth. Despite our attempts, commercial mammalian ECM components do not seem to replicate D17 ECM, and D17 ECM is not competent to convey cell spreading or motility to nonmotile S2 or S2R + cells. D17 ECM is essential for culturing D17 cells on glass surfaces for wound healing assays, single cell motility or fluorescent visualization. Although the above-mentioned assays can be performed in tissue culture-treated plastic vessels, the use of glass surfaces allows greater resolution and decreased chromatic aberrations. One alternative is using a tissue culture- 
treated optical polystyrene that does not require ECM coating and can be used for oil immersion microscopy.

Because the concentration of ECM components can vary from batch to batch, it is important that investigators test newly made ECM to ensure consistency across many experiments. To do this, investigators should plate and image D17 cells at a range of ECM concentrations (diluted in PBS, see Step 15, generally ranging from 1:10 to 1:300). Analyzing the velocity and other parameters of migration (see Step 23) for each concentration should allow investigators to find an ECM concentration that either replicates results from a previous ECM batch or has the desired parameters of migration for a given experimental approach.

Transient transfection-D17 transfection will allow the user to image the dynamics and localization of exogenous transgenes through high-resolution immunofluorescence, or in real time using bioluminescent tags such as enhanced green fluorescent protein. D17 cells are amenable to transfection with most of the expression constructs currently used for S2 cells. One caveat is that D17 cells seem to be somewhat insensitive to the commonly used metallothionein promoter constructs (referred to as pMT vectors). These reagents can be used, but require substantially higher doses of copper sulfate to induce gene expression (between $500 \mu \mathrm{M}$ and $5 \mathrm{mM}$ concentrations). On the basis of our experience, constitutive promoters such as the actin promoter or the OPIE2 promoter give medium to high levels of expression and can be used when an endogenous promoter is unavailable. It is important that researchers use the proper controls to both ensure the expression of their desired transgene and to account for deleterious effects on D17 cell viability and cell migration. Expressing the empty vector backbone of a given transgene that only expresses an affinity or bioluminescent tag in D17 cells can both troubleshoot problems with the transfection protocol and control for the effects of toxic gene expression with a given assay. Users can also test transgenes by transfection in $\mathrm{S} 2$ cells or other nonmotile Drosophila cell lines to determine the effects of their transgene on cell morphology. Using this protocol we normally achieve 10-40\% transfection efficiency in D17 cells after $48 \mathrm{~h}$. The transfection protocol outlined here differs from protocols for transecting S2 cells, by which we can commonly achieve $20-70 \%$ transfection efficiency, but both cell types seem to respond poorly to the transfection protocol of the other cell type.

Migration assays-The ability to assay cell migration is the key strength of designing experiments using the D17 cell line. Here we outline a classical wound healing assay that has been previously used to measure the migratory capability of mammalian cells ${ }^{27}$. One of the key advantages of this kind of assay is that it can easily be scaled from the examination of single conditions to high-throughput assays of many dsRNA treatments or conditions ${ }^{28}$. We previously used this method to initially determine that depletion of the micro-tubulesevering protein, katanin, leads to an increase in the rate of wound closure ${ }^{22}$. We then went on to examine the migration parameters of single cells migrating at subconfluent densities. This twofold approach allowed us to determine an initial phenotype before further defining the exact mechanism of this migratory gain of function ${ }^{22}$. In addition, investigators can often combine the two techniques (as described in Step 22) to measure both wound closure as well as the migratory properties of single cells moving into the wound. 
When imaging D17 cells as single cells or within the context of a scratch wound assay, it is important that investigators consider: (i) the resolution of observation, (ii) the temporal resolution of time series acquisition and (iii) the maintenance of consistency between conditions.

The magnification used for acquiring images of D17 migration should encompass the entire wound when using a scratch wound assay. When tracking single cells, the resolution for image acquisition should also be adequate to provide the necessary contrast and resolution to faithfully track cells as well as determine differences in morphology. We recommend using between $\times 10$ and $\times 20$ magnification for the scratch wound assay, and increasing magnification to between $\times 20$ and $\times 40$ when imaging and tracking single D17 cells.

Users should be aware of the spatial resolution they are using to follow D17 migration. During a scratch wound assay, users should empirically determine, on the basis of the size of their scratch, a time frame in which it would be feasible to detect both loss- and gain-offunction migration phenotypes. This often means setting a final time point where controltreated cells migrate into the wound but do not completely fill the wound area.

When creating a time-lapse series of wound healing or of sub-confluent, single D17 cells, users should ensure that the rate of image acquisition is adequate to capture all cell movements. Lengthy intervals might lose small movements of cells and potentially skew measurements of directionality and velocity. This should be balanced by limiting the cell's exposure to heat and evaporation caused by light illumination. Typically, intervals of 3-7 $\min$ should be an adequate rate of acquisition to alleviate these effects.

Finally, users should attempt to maintain consistent assay conditions across various experimental conditions. This includes the number of cells treated and plated for each condition, although this can difficult to achieve when treatments affect cell viability. Performing a wound healing assay with a pipette tip can also cause inconsistencies between conditions. Users should attempt to create the most uniform scratches possible between wells by maintaining a constant angle and pressure on the pipette tip while wounding cell monolayers. Custom and commercial apparatus can also be used to aid in wounding multiwell plates of D17 cells $^{28,29}$.

At present, one of the primary limitations of using the D17 cell line is the lack of directional migration assays toward chemotatic, haptotatic or durotatic cues. Analysis of D17 migration using a scratch wound assay or imaging single cells migrating in culture is currently restricted to the nondirectional migration of cells on the basis of our lack of known guidance cues. Because the migration of D17 cells is random, it can also lead to heterogeneity in the total number of cells in a population that are migrating as well as the persistence of migration in a single direction and the duration of migration. Investigators should be aware of this limitation and repeat experiments at least three times, sampling an adequate number of cells to truly determine the effect of dsRNA or other treatment on a population of cells. Understanding the cues that govern D17 motility will be an important advance to harness these guidance cues to spatially and temporally direct D17 migration. 


\section{MATERIALS}

REAGENTS

- ML-DmD17-c3 cell stocks are available from the DGRC (http://dgrc.cgb.indiana.edu/)

- Schneider's insect medium (Invitrogen, cat. no. 11720-034)

- Antibiotic/antimycotic (Invitrogen, cat. no. 15240-096)

- FBS (Invitrogen, cat. no. 10099-141)

- Human insulin (Invitrogen, cat. no. 12585-014) C CRITICAL Many vendors sell FBS that is already heat-inactivated, usually at temperatures exceeding $65^{\circ} \mathrm{C}$. This heatinactivated serum is not suitable for D17 cell culture, as it does not support long-term cell passage, presumably because of the inactivation of important trophic factors. Non-heatinactivated serum can be purchased and heat-inactivated by submerging the thawed serum in a $55{ }^{\circ} \mathrm{C}$ water bath for $1 \mathrm{~h}$ with occasional inversions to mix the serum.

- Cell dissociation buffer, PBS based (Invitrogen, cat. no. 13151-014)

- D17 growth medium (see REAGENT SETUP) C CRITICAL D17 growth medium should be made in advance of receiving frozen ampules from the Bloomington stock center.

- Sulfuric acid (Fisher Scientific, cat. no. SA212-1) CAUTION Caustic reagent.

- Hydrogen peroxide (Fisher Scientific, cat. no. H325-100) CAUTION Irritant.

- Sterile PBS (Invitrogen, cat. no. 10010-023)

- Saturated ammonium sulfate solution (Sigma-Aldrich, cat. no. A6387; see REAGENT SETUP) CAUTION Irritant. Users should wear gloves when handling ammonium sulfate.

- Conditioned D17 growth medium (see Box 1)

- Fugene HD transfection reagent (Roche, cat. no. 04709705001)

- Sterile water

- Suitable transfection vectors, e.g., metallothionein promotor (pMT vectors; Invitrogen, cat. no. V4120-20), OpIE2 promoter pIZ vectors (Invitrogen, cat. no. V800001) or actin promoter pAc5.1 vectors (Invitrogen, cat. no. V4110-20). Vector backbones can be modified or purchased to accommodate bioluminescent probes and other user-specific transgenes.

- D17 freezing medium (see REAGENT SETUP)

$\cdot$ DMSO

- Distilled, deionized water 
- Ethanol

- dsRNA (these are user-defined and created using standard methods ${ }^{21}$ )

\section{EQUIPMENT}

- UV transilluminator (such as Fisher Scientific, cat. no. PBDLT88AQ) or other power source such as a plasma treater that can bombard treated cover slips with at least $8,000 \mu \mathrm{w}$ $\mathrm{cm}^{-2}$ CAUTION Always use appropriate eye and skin apparatus and necessary shielding when using UV light.

- Polystyrene Petri dishes (35 mm × $10 \mathrm{~mm}$; Becton Dickinson, cat. no. 351008)

- Glass-bottom multiwell plate (Greiner, cat. no. 662892)

- Optical grade polystyrene/polymer base multiwell plate (Nunc, cat. no. 165305)

- Glass cover slips, no. 1.5, $22 \mathrm{~mm}^{2}$ (Corning, cat. no. 2940-225)

- Cover slip rack, porcelain (Coors, through Thomas Scientific, cat. no. 8542E40)

- Swinging-bucket tabletop centrifuge

- Tissue culture flask vessels ( $25 \mathrm{~cm}^{2}$, T25; Becton Dickinson, cat. no. 353082)

- Tissue culture flask vessels ( $75 \mathrm{~cm}^{2}$, T75; Becton Dickinson, cat. no. 353135 )

- Sterile, laminar flow hood

- Sterile bottle-top filter flask (500 ml; Corning, cat. no. 431117)

- Cell scraper (25 cm; Becton Dickinson, cat. no. 353086)

- Dialysis tubing (Pierce, cat. no. 68035)

- Dialysis clips (Pierce, cat. no. 68011)

- Inverted microscope with charge-coupled device camera

- Microscope image acquisition software, e.g., MetaMorph (Molecular Devices) or NIS Elements (Nikon)

- ImageJ software (http://rsbweb.nih.gov/ij/) and Manual Tracking plugin (Fabrice Cordelières, http://rsbweb.nih.gov/ij/plugins/track/track.html)

\section{EQUIPMENT SETUP}

Cover slip preparation-Before ECM-treating glass cover slips, cover slips should be cleaned by immersion in a strong acid for at least $1 \mathrm{~h}$. Before treatment, load untreated cover slips into porcelain racks. To prepare the acid solution, add two parts sulfuric acid to one part hydrogen peroxide. Typically, this volume is $200 \mathrm{ml}$ of sulfuric acid added to $100 \mathrm{ml}$ of 
hydrogen peroxide within a 1 liter glass beaker. Carefully lower the porcelain rack(s) into the acid solution and ensure that the cover slips are fully immersed. After 1-h acid treatment, cover slips should be rinsed continuously with sterile running water for $15 \mathrm{~min}$ and dried. Once dry, place in 35-mm dishes either individually or together to prevent dust accumulation on their surface.

\section{REAGENT SETUP}

D17 growth medium-Prepare growth medium (cell culture medium) within a sterile, laminar flow hood by combining $5 \mathrm{ml}$ of antibiotic/antimycotic (100x stock concentration), $50 \mathrm{ml}$ of heat-inactivated FBS (10\% total medium volume), $1.25 \mathrm{ml}$ of human insulin (4 mg $\mathrm{ml}^{-1}$ stock concentration to final $10 \mu \mathrm{g} \mathrm{ml}{ }^{-1}$ final concentration) and $445 \mathrm{ml}$ of Schneider's medium to a final volume of $500 \mathrm{ml}$. This solution should then be sterile filtered using a 2$\mu \mathrm{m}$ filter flask to exclude any precipitated serum or contaminates. Store at $4{ }^{\circ} \mathrm{C}$ and warm to room temperature $\left(\sim 25^{\circ} \mathrm{C}\right)$ before use. D17 growth medium is stable for 1-3 months, although serum crystals will form over time. These crystals should remain on the bottle bottom and do not seem to affect D17 culture.

D17 freezing medium-D17 freezing medium should be freshly made before each use by mixing $4 \mathrm{ml}$ of conditioned D17 growth medium, $4 \mathrm{ml}$ of fresh D17 growth medium and 1 $\mathrm{ml}$ of sterile DMSO.

Saturated ammonium sulfate solution-Prepare a saturated ammonium sulfate solution by adding $\sim 750 \mathrm{~g}$ of ammonium sulfate to 1 liter of distilled and deionized water. Heat the solution until the ammonium sulfate is totally dissolved and then cool to room temperature $\left(25^{\circ} \mathrm{C}\right)$. Upon cooling, crystals should form, indicating the solution is indeed saturated. The saturated ammonium sulfate can be stored and used at room temperature and has an indefinite shelf life.

\section{PROCEDURE}

\section{Thawing D17 cells TIMING $1.5-2.5 \mathrm{~h}$}

1 In a sterile laminar flow hood, add $7 \mathrm{ml}$ of D17 growth medium to a T25 tissue culture flask; this should be carried out before thawing the cells.

- CRITICAL STEP Because the delivery conditions of dry ice are suboptimal for the storage of frozen cells, it is important that the ampule of cells be plated as soon as it arrives.

2 Remove the vial of cells from the dry ice delivery container and immerse the bottom of the ampule in a $37{ }^{\circ} \mathrm{C}$ water bath, shaking often to thaw the ampule as quickly as possibly to room temperature.

- CRITICAL STEP All attempts should be made to keep the cryovial lid from coming into contact with the water bath water. This will prevent contamination of the D17 cells.

3 Once the ampule contents are liquid, remove from the water bath and sterilize the ampule by wiping the outside with a $70 \%$ (vol/vol) ethanol solution. 
4 In the laminar flow hood, add the contents of the ampule to the $7 \mathrm{ml}$ of D17 growth medium in the T25 culture flask prepared in Step 1.

5 Leave the cells for $1-2 \mathrm{~h}$ at room temperature $\left(25^{\circ} \mathrm{C}\right)$ to attach and spread on the bottom of the culture flask.

6 Remove the medium and replace it with a fresh $7 \mathrm{ml}$ of growth medium to remove any DMSO that was present in the frozen cell solution. Grow D17 cultures in a sterile incubator or on a shelf at $25^{\circ} \mathrm{C}$. Flasks do not require gas exchange vents or special atmospheric considerations.

\section{Passaging D17 cultures TIMING 20 - 60 min}

7 Remove the D17 growth medium from the culture flask and store this conditioned medium at $4{ }^{\circ} \mathrm{C}$ for ECM production (see Box 1).

8 Split D17 cells using either cell dissociation buffer (option A) or using a cell scraper (option B).

(A) Passaging using dissociation buffer

(i) Wash the cells in the culture vessel by gently pipetting $2 \mathrm{ml}$ of cell dissociation buffer onto the cells, briefly tilting the vessel to allow the buffer to wash over the cells, and then removing the buffer with a pipette from the tilted culture vessel.

(ii) Add another $2 \mathrm{ml}$ of cell dissociation buffer, or enough volume to cover the bottom of the flask. Incubate the cells for 25-30 min at room temperature outside the laminar flow hood. Under magnification, the cells should be seen to round up and lose cell-cell adhesion. ? TROUBLESHOOTING

(iii) Gently remove the cell dissociation buffer with a pipette and add $5 \mathrm{ml}$ of fresh D17 growth medium. Vigorously pipette the medium up and down several times to detach the cells from the bottom of the culture flask.

(B) Passaging using a cell scraper

(i) Add $6 \mathrm{ml}$ of fresh D17 growth medium to the flask. Insert the cell scraper into the flask and move the blade across the flask bottom, trying to remove a maximum amount of cells with the fewest blade strokes.

9 Seed the cell suspension into new culture vessels at concentrations of 1 to $2 \times 10^{6}$ cells per $\mathrm{ml}$ or roughly $40-60 \%$ confluency in either a T25 or T75 flask. The D17 cells should reach $100 \%$ confluency after 3-7 d depending on the concentration of cells seeded. Within the culture flask, the cells should reach a monolayer with small foci of additional cell growth above the monolayer. In cases where the cells are grown from sparse conditions, the user may find large colonies of cells that form islands of monolayers with exaggerated foci of cells on top. This is also adequate to passage to new vessels, as the cells contained within the foci should be competent to repopulate a new vessel. Aliquots of D17 cells can be frozen in D17 freezing medium using previously published protocols for S2 cells ${ }^{21}$. 
- CRITICAL STEP The growth rate and survival of D17 cells is very sensitive to plating density. The most common error when culturing D17 is to plate them at low densities, which can result in extremely slow growth or cell death. An optimal density of $40-60 \%$ is essential for healthy cells and confluent passaging between 3 and $6 \mathrm{~d}$.

- CRITICAL STEP The user should keep track of passage iterations, as D17 cells will reach a terminal state after 25-30 passages, at which point they may respond poorly to RNAi or transfection and may have slow growth or abnormal morphology. ?

TROUBLESHOOTING

\section{RNAi treatment $\odot$ TIMING 7-9 d}

10 Plate cells in tissue culture-treated multiwell culture vessel at subconfluent density (40$60 \%$ or $4,000-5,000$ cells $\mathrm{mm}^{-1}$ ). We recommend performing RNAi in tissue culturetreated vessels and then transferring cells to cover slips or glass vessels, when possible.

11 Allow the cells to adhere to the vessel bottom for $1 \mathrm{~h}$ at room temperature $\left(25^{\circ} \mathrm{C}\right)$. If desired, cells can be transfected (see Box 2) within 30 min after plating and at anytime during RNAi treatment.

12 Remove medium and replace with culture medium containing $\sim 10 \mu \mathrm{g} \mathrm{ml}^{-1}$ dsRNA. Treated cells should be incubated as normally passaged cells at room temperature $\left(25^{\circ} \mathrm{C}\right)$.

13 Repeat dsRNA treatment (Step 11) every $2 \mathrm{~d}$ for $7-9 \mathrm{~d}$ of treatment.

14 At 6-9 d of dsRNA treatment, harvest cell lysates for western blot analysis or transfer to ECM-coated vessels (Steps 15-18) to assay for loss-of-function phenotypes; for example, migration phenotypes can be assayed as described in Steps 19-22 below. The user should empirically determine the optimal length of treatment for each dsRNA construct and gene product. ? TROUBLESHOOTING

\section{Plating D17 cells on ECM-coated surfaces $\bigcirc$ TIMING 1 h}

15 Use a stock solution of D17 cell ECM (see Box 1) to dilute the ECM solution in sterile PBS to the desired concentration. The exact concentration of ECM will need to be determined empirically by the user on the basis of the optimal assay conditions and the yield of ECM purified per batch (see EXPERIMENTAL DESIGN). As a general starting point, dilutions of ECM to PBS ranging from 1:50 to 1:200 seem to support robust attachment and migration in our hands. ? TROUBLESHOOTING

16 Apply enough of the ECM/PBS solution to cover the surface of the cover slip or glassbottom plate intended for culture.

17 Cross-link the ECM solution to the glass surface by plasma treatment for several minutes or high-energy UV treatment for $45 \mathrm{~min}$. For many users, placing the dish or cover slip onto the surface of a UV transilluminatior used to visualize ethidium bromide-stained agarose gels is sufficient, provided it can produce enough energy $\left(\sim 8,000 \mu \mathrm{w} \mathrm{cm}^{-2}\right)$ to cross-link the ECM to the glass surface. 
- CRITICAL STEP D17 cells should be plated within several hours of ECM crosslinking. Plasma or UV treatment is a temporary cross-linking procedure, which can affect the efficiency of D17 adherence and motility for some applications. ?

\section{TROUBLESHOOTING}

18 After cross-linking, remove the ECM/PBS solution. Plate the cells at the desired density to produce an adherent monolayer or single cells. D17 cells should adhere and spread on the cross-linked glass surface within $1 \mathrm{~h}$. Cells are viable on these cross-linked surfaces for several days and can proliferate to form confluent monolayers if plated in subconfluent number. Generally, for subconfluent plating, cell densities of 4,000-5,000 cells per mm of surface are sufficient, or densities of 7,000-8,000 cells per mm when cell monolayers are desired.

\section{Wound migration assay 0 TIMING 18-24 h}

19 Seed cells at monolayer densities on either tissue culture-treated vessels or ECM-coated glass surfaces at room temperature $\left(25^{\circ} \mathrm{C}\right)$. Cells can be allowed to settle anywhere from 2 to $24 \mathrm{~h}$ before wounding cell monolayers. Additionally, if too few cells are initially seeded, users can apply additional cells to increase the confluency of D17 cells to monolayer density.

20 Once monolayers are obtained, use a $200-\mu l$ pipette tip or other pointed implement to scratch though the monolayer, creating a wound. Wounds should be at least $200 \mu \mathrm{m}$ in size without visible obstructions of cell debris or substrate etching.

21 Once wounded, pipette off the growth medium and wash the wells with fresh medium to remove debris.

22 Mount the vessel on an inverted light microscope with phase-contrast or differential interference contrast filters and begin imaging. D17 wounded monolayers can be imaged over the course of 18-24 h using either discrete time points (option A) or a time-lapse series (option B).

(A) Imaging D17 wounded monolayers using discrete time points

(i) Keep the vessel stationary on the inverted microscope throughout the migration assay and acquire images of the wound at regular intervals; alternatively, acquire a set of images representing the beginning and end of the migration assay.

(ii) After acquiring all time point images, use ImageJ software to outline the scratch area and measure the area of the wound at each time point. Compare the total area between the initial time point and later time points to obtain the percentage of wound closure over a given time.

(B) Imaging D17 wounded monolayers using a time-lapse series

(i) Keep the vessel stationary on the inverted microscope throughout the course of the assay, and acquire images at regular intervals of between 3 and $7 \mathrm{~min}$ (see EXPERIMENTAL DESIGN). 
(ii) Use ImageJ software to assemble time-lapse intervals into a multi-image tiff file.

(iii) Apply the Manual Tracking plug-in to the multi-image tiff files to track individual cells as they migrate into the wounded area. Parameters such as instantaneous and mean velocity, distance and directionality can be obtained using this plug-in. Analysis of D17 single-cell motility at subconfluent densities can also be performed using Steps 22B(i-iii).

? TROUBLESHOOTING

Troubleshooting advice can be found in Table $\mathbf{1}$.

TIMING

EQUIPMENT SETUP, Cover slip preparation: $\sim 1.5 \mathrm{~h}$

REAGENT SETUP, D17 growth medium: $\sim 15$ min once all ingredients are thawed

REAGENT SETUP, Saturated ammonium sulfate: $~ 30 \mathrm{~min}$

Steps 1-6, Thawing D17 cells: $1.5-2.5 \mathrm{~h}$

Steps 7-9, Passaging cells: 20-60 min

Step 8(A), Passaging using cell dissociation buffer: $25-60 \mathrm{~min}$

Step 8(B), Passaging cell scraper: 20 min

Steps 10-12, RNAi plating and initial treatment: $1.5 \mathrm{~h}$

Step 13, Subsequent dsRNA treatment: $30 \mathrm{~min}$

Step 14, Full dsRNA treatment: 7-9 d Steps 15-18, Plating D17 cells on ECM surfaces: $1 \mathrm{~h}$

Steps 19-22, Wound healing assay, 18-24 h; can be performed concurrently with the end of dsRNA treatment

Box 1, Preparing D17 ECM: 18-24 h

Box 2, D17 transient transfection, $30 \mathrm{~min}$; can be performed concurrently with dsRNA treatment, wound healing treatment or both

\section{ANTICIPATED RESULTS}

Using either a wound healing assay (Fig. 1a and Supplementary Video 2) or by imaging single cells (Fig. 2) over various time periods, the investigator can acquire time-lapse data sets from which they may derive various parameters of cell migration, including distance, velocity (Fig. 1b) and directional persistence. In a wound healing assay, an investigator can expect control-treated cells to migrate from both sides, not necessarily as a single-cell sheet, to fill in approximately $150-250 \mu \mathrm{m}$ of the wound area over $18-24 \mathrm{~h}$. 
By optimizing the duration of dsRNA treatment to obtain greater than $85 \%$ knockdown by western blot analysis, the user should expect a highly penetrant RNAi migration phenotype. Combining transfection of D17 cells with a wound healing assay or single-cell migration assay will also allow the user to determine the effects of overexpression on cell migration. Investigators can also take advantage of dsRNA against the 5[.radicalex] or 3[.radicalex] untranslated region of an endogenous gene, allowing them to express exogenous cDNA transgenes to examine the sufficiency of a particular gene to rescue RNAi migration phenotypes. In these cases, the lower transfection efficiency of 10-40\% expressing cells can be advantageous, as the user can use untransfected cells within the same condition as controls.

\section{Supplementary Material}

Refer to Web version on PubMed Central for supplementary material.

\section{Acknowledgments}

We thank members of the Rogers lab for helpful discussions regarding the application of this protocol. We would also like to acknowledge M. Peifer for the generous gift of Canoe antibody and the DGRC for cell lines and helpful protocols.

\section{References}

1. Schneider I. Cell lines derived from late embryonic stages of Drosophila melanogaster. J. Embryol. Exp. Morphol. 1972; 27:353-365. [PubMed: 4625067]

2. Yanagawa S, Lee JS, Ishimoto A. Identification and characterization of a novel line of Drosophila Schneider S2 cells that respond to wingless signaling. J. Biol. Chem. 1998; 273:32353-32359. [PubMed: 9822716]

3. Clemens JC, et al. Use of double-stranded RNA interference in Drosophila cell lines to dissect signal transduction pathways. Proc. Natl. Acad. Sci. USA. 2000; 97:6499-6503. [PubMed: 10823906]

4. Rogers SL. Drosophila EB1 is important for proper assembly, dynamics, and positioning of the mitotic spindle. J. Cell. Biol. 2002; 158:873-884. [PubMed: 12213835]

5. Ramadan N, Flockhart I, Booker M, Perrimon N, Mathey-Prevot B. Design and implementation of high-throughput RNAi screens in cultured Drosophila cells. Nat. Protoc. 2007; 2:2245-2264. [PubMed: 17853882]

6. Adams MD, et al. The genome sequence of Drosophila melanogaster. Science. 2000; 287:21852195. [PubMed: 10731132]

7. Rogers GC, Rusan NM, Peifer M, Rogers SL. A multicomponent assembly pathway contributes to the formation of acentrosomal microtubule arrays in interphase Drosophila cells. Mol. Biol. Cell. 2008; 19:3163-3178. [PubMed: 18463166]

8. Wheeler SR, et al. Neurexin IV and Wrapper interactions mediate Drosophila midline glial migration and axonal ensheathment. Development. 2009; 136:1147-1157. [PubMed: 19270173]

9. Johnston CA, Hirono K, Prehoda KE, Doe CQ. Identification of an Aurora-A/PinsLINKER/Dlg spindle orientation pathway using induced cell polarity in S2 cells. Cell. 2009; 138:1150-1163. [PubMed: 19766567]

10. Jiang L, Rogers SL, Crews ST. The Drosophila Dead end Arf-like3 GTPase controls vesicle trafficking during tracheal fusion cell morphogenesis. Dev. Biol. 2007; 311:487-499. [PubMed: 17919535]

11. Dorer MS, Kirton D, Bader JS, Isberg RR. RNA interference analysis of Legionella in Drosophila cells: exploitation of early secretory apparatus dynamics. PLoS. Pathog. 2006; 2:e34. [PubMed: 16652170] 
12. Wagner EJ, et al. A genome-wide RNA interference screen reveals that variant histones are necessary for replication-dependent histone pre-mRNA processing. Mol. Cell. 2007; 28:692-699. [PubMed: 18042462]

13. D'Ambrosio MV, Vale RD. A whole genome RNAi screen of Drosophila S2 cell spreading performed using automated computational image analysis. J. Cell. Biol. 2010; 191:471-478. [PubMed: 21041442]

14. Ui K, Ueda R, Miyake T. Cell lines from imaginal discs of Drosophila melanogaster. In Vitro Cell. Dev. Biol. 1987; 23:707-711. [PubMed: 3117765]

15. Wood W, Faria C, Jacinto A. Distinct mechanisms regulate hemocyte chemotaxis during development and wound healing in Drosophila melanogaster. J. Cell. Biol. 2006; 173:405-416. [PubMed: 16651377]

16. Wood W, Jacinto A. Drosophila melanogaster embryonic haemocytes: masters of multitasking. Nat. Rev. Mol. Cell. Biol. 2007; 8:542-551. [PubMed: 17565363]

17. Celniker SE, et al. Unlocking the secrets of the genome. Nature. 2009; 459:927-930. [PubMed: 19536255]

18. Rehorn KP, Thelen H, Michelson AM, Reuter R. A molecular aspect of hematopoiesis and endoderm development common to vertebrates and Drosophila. Development. 1996; 122:40234031. [PubMed: 9012522]

19. Cherbas L, et al. The transcriptional diversity of 25 Drosophila cell lines. Genome Res. 2011; 21:301-314. [PubMed: 21177962]

20. Fasano L, et al. The gene teashirt is required for the development of Drosophila embryonic trunk segments and encodes a protein with widely spaced zinc finger motifs. Cell. 1991; 64:63-79. [PubMed: 1846092]

21. Rogers SL, Rogers GC. Culture of Drosophila S2 cells and their use for RNAi-mediated loss-offunction studies and immunofluorescence microscopy. Nat. Protoc. 2008; 3:606-611. [PubMed: 18388942]

22. Zhang D, et al. Drosophila katanin is a microtubule depolymerase that regulates corticalmicrotubule plus-end interactions and cell migration. Nat. Cell. Biol. 2011; 13:361-370. [PubMed: 21378981]

23. Drechsel DN, Hyman AA, Hall A, Glotzer M. A requirement for Rho and Cdc42 during cytokinesis in Xenopus embryos. Curr. Biol. 1997; 7:12-23. [PubMed: 8999996]

24. Paladi M, Tepass U. Function of Rho GTPases in embryonic blood cell migration in Drosophila. J. Cell. Sci. 2004; 117:6313-6326. [PubMed: 15561773]

25. Stramer B. Live imaging of wound inflammation in Drosophila embryos reveals key roles for small GTPases during in vivo cell migration. J. Cell. Biol. 2005; 168:567-573. [PubMed: 15699212]

26. Fessler JH, Nelson RE, Fessler LI. Preparation of extracellular matrix. Methods Cell Biol. 1994; 44:303-328. [PubMed: 7707960]

27. Liang C, Park AY, Guan J. In vitro scratch assay: a convenient and inexpensive method for analysis of cell migration in vitro. Nat. Protoc. 2007; 2:329-333. [PubMed: 17406593]

28. Yarrow JC, Perlman ZE, Westwood NJ, Mitchison TJ. A high-throughput cell migration assay using scratch wound healing, a comparison of image-based readout methods. BMC Biotechnol. 2004; 4:21. [PubMed: 15357872]

29. Vitorino P, Meyer T. Modular control of endothelial sheet migration. Genes Dev. 2008; 22:32683281. [PubMed: 19056882] 


\section{BOX 1 PREPARING D17 EXTRACELLULAR MATRIX — TIMING 18-24 H}

1. Collect conditioned medium from confluent flasks of adherent D17 cells and store at 4 ${ }^{\circ} \mathrm{C}$. This can be collected every $3 \mathrm{~d}$ from $100 \%$ confluent flasks or every $6 \mathrm{~d}$ from subconfluent flasks. The most straightforward approach is to save the medium that cells have been cultured in when passaging cells to new culture vessels.

2. Once a sufficient amount has been collected (at least $100 \mathrm{ml}$ ), spin the medium at $200 \mathrm{~g}$ for $10 \mathrm{~min}$ to remove any cells or large precipitants.

CRITICAL STEP Because there is some variability between lots of prepared ECM, it is important in an effort to maintain consistency that a large enough batch of conditioned medium is processed to accommodate the user's aims. To this end, we would recommend processing between 200 and $400 \mathrm{ml}$ of conditioned medium at any one time, which will produce enough ECM to last the user for more than 100 experiments, depending on the final concentration at which it is used.

3. Pour the cell-free medium into a glass beaker and stir slowly on a stir plate.

4. Add $\sim 40 \%$ volume of saturated ammonium sulfate dropwise to the slowly stirring medium (i.e., $40 \mathrm{ml}$ ammonium sulfate added to $100 \mathrm{ml}$ conditioned medium). The key to success at this step is to continue adding ammonium sulfate until the medium is cloudy and opaque (which indicates the precipitation of large proteins out of solution) rather than adding a precise volume.

5. Pour the precipitated medium solution into 50-ml conical tubes and spin at speeds in excess of $3,000 \mathrm{~g}$ for $30 \mathrm{~min}$ to $1 \mathrm{~h}$ at $4{ }^{\circ} \mathrm{C}$. After centrifugation, the precipitated proteins should be pelleted and the supernatant should again be clear.

6. Discard the supernatant and resuspend the pellet in 1/100th of the original medium volume (i.e., for $100 \mathrm{ml}$ of conditioned medium, resuspend the pellet in $1 \mathrm{ml}$ ) in cold, sterile PBS.

7. Pipette the resuspend pellet into a dialysis bag and dialyze in 1 liter of PBS at $4{ }^{\circ} \mathrm{C}$, changing the PBS out 2-3 times over the course of 16-24 h to remove the ammonium sulfate from the ECM solution.

PAUSE POINT The resuspended ECM pellet can be left to dialyze in fresh PBS overnight at $4{ }^{\circ} \mathrm{C}$.

8. Aliquot the dialyzed ECM into 1.7-ml microcentrifuge tubes and spin in a tabletop centrifuge at $4{ }^{\circ} \mathrm{C}$ at speeds in excess of $16,000 \mathrm{~g}$ for $10 \mathrm{~min}$ and transferring the supernatant to another microcentrifuge tube to remove any remaining precipitate.

9. The ECM is now ready for use (see Steps $15-18$ of the main PROCEDURE). Test each new batch for efficacy to match the ideal assay conditions (see EXPERIMENTAL DESIGN).

- PAUSE POINT ECM can be stored short-term (1-2 months) at $4{ }^{\circ} \mathrm{C}$ or long-term by flash-freezing it in liquid nitrogen and storing at $-80{ }^{\circ} \mathrm{C}$. 


\section{BOX 2 TRANSIENT TRANSFECTION OF D17 CELLS ○ TIMING 30 MIN}

1. Plate D17 cells in either an ECM-coated glass vessel or a tissue culture-treated polystyrene multiwell dish to a confluency of $50-80 \%$ and leave them to adhere at room temperature for $30 \mathrm{~min}$ to $24 \mathrm{~h}$.

2. After the cells have adhered to the culture vessel surface, replace the medium to remove cell debris.

3. Make up the Fugene HD transfection mixture and treat cells according to the manufacturer's protocol at a ratio of $3 \mu \mathrm{l}$ Fugene HD to $2 \mu \mathrm{g}$ total DNA (3:2) diluted in sterile water, although we recommend that users empirically determine their optimal transfection conditions for each transgene. The transfection reagent can remain on the plated D17 cells indefinitely.

4. Assess gene expression; this can be carried out within $24 \mathrm{~h}$ depending on the type of promoter used to induce transgene expression.

\section{? TROUBLESHOOTING}





Figure 1.

D17-c3 (D17) is a motile Drosophila melanogaster cell line. (a) D17 cells plated to monolayer confluency and scratched with a $200 \mu \mathrm{l}$ pipette tip (left) and allowed to migrate into the wounding area over $16 \mathrm{~h}$ (right). Representative migration tracks from two cells are indicated on the right in blue and green. The full movie can be found in Supplementary Video 2. (b) Measure of instantaneous or step velocity of individual D17 cells from either a control or SCAR dsRNA treatment; $n=3,10$ cells tracked for each experiment. Error bars indicate s.e.m. (c) D17 cells treated with SCAR dsRNA and wounded at $0 \mathrm{~h}$ (top) and after $16 \mathrm{~h}$ (bottom). Inhibition of Arp2/3-mediated actin nucleation by depletion of its activator SCAR significantly inhibits D17 cell motility. (d) D17 cells after $7 \mathrm{~d}$ of treatment with either control dsRNA (left) or Rho dsRNA (right). Depletion of Rho results in cytokinesis defects and multinucleate cells. (e) Immunoblots of S2 or D17 lysates for tubulin (DM1a, Sigma) or Rho1 (p1D9, Developmental Hybridoma Bank, University of Iowa). Lysates were taken from cells treated with control dsRNA or Rho1 dsRNA for $7 \mathrm{~d}$, and represent equal protein load as determined by Bradford assay. 


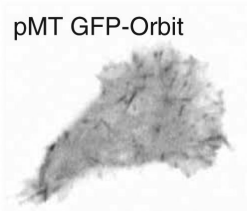

$10 \mu \mathrm{m}$
$0 \mathrm{~s}$

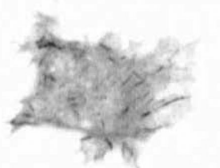

$72 \mathrm{~s}$

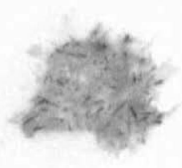

$24 \mathrm{~s}$

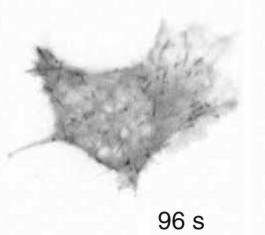

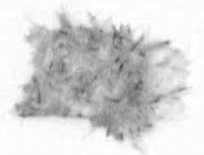

$48 \mathrm{~s}$

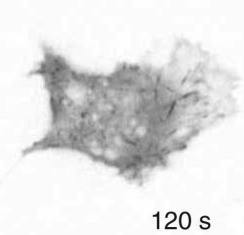

Figure 2.

Dynamic protein localization within a migrating D17 cell. D17 cell expressing the Drosophila microtubule plus-end protein, Orbit/Mast, tagged with GFP under the metallothionein promoter (pMT GFP-Orbit) imaged by spinning disc confocal microscopy. The full movie is available as Supplementary Video 1. 


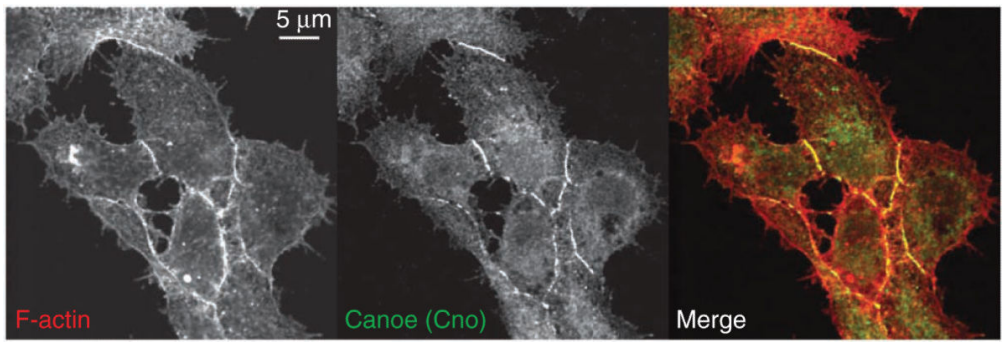

Figure 3.

Immunofluorescence localization of cell-cell junction protein Canoe (Cno) in D17 cells. D17 cell stained for F-actin (left) using Alexa 488 phalloidin (Molecular Probes) and the junctional protein Canoe (Cno; middle). Canoe localizes specifically to areas of cell-cell contact. 


\section{TABLE 1}

| Troubleshooting table.

\begin{tabular}{|c|c|c|c|}
\hline Step & Problem & Possible reason & Solution \\
\hline $8 \mathrm{~A}(\mathrm{ii})$ & $\begin{array}{l}\text { D17 cells do not detach after } \\
\text { treatment with cell } \\
\text { dissociation buffer }\end{array}$ & $\begin{array}{l}\text { D17 growth medium may remain after } \\
\text { washing with dissociation buffer or D17 } \\
\text { cells are present in high density and are } \\
\text { more resistant to dissociation buffer }\end{array}$ & $\begin{array}{l}\text { Incubate D17 cells for longer periods with cell } \\
\text { dissociation buffer. Use a more powerful } \\
\text { electronic pipette to mechanically remove cells }\end{array}$ \\
\hline \multirow[t]{2}{*}{9} & $\begin{array}{l}\text { D17 cultures die after a small } \\
\text { number of initial passages }\end{array}$ & $\begin{array}{l}\text { Lack of growth factors in the medium can } \\
\text { lead to a death of D17 cells over the course } \\
\text { of several passages. This can be caused by } \\
\text { an improper inactivation of FBS }\end{array}$ & $\begin{array}{l}\text { Be sure that FBS is heat inactivated at a } \\
\text { temperature of } 55^{\circ} \mathrm{C} \text { (see MATERIALS) }\end{array}$ \\
\hline & $\begin{array}{l}\text { D17 cells never reach } 100 \% \\
\text { confluency, but instead form } \\
\text { large foci and single cells } \\
\text { adopt a spindly morphology }\end{array}$ & $\begin{array}{l}\text { D17 cells were possibly plated at too low a } \\
\text { density }\end{array}$ & $\begin{array}{l}\text { Consider dissociating and passaging the foci with } \\
\text { vigorous pipetting or dissociation buffer into a } \\
\text { smaller culture flask. Consider replacing half the } \\
\text { culture medium with fresh D } 17 \text { growth medium to } \\
\text { provide fresh growth factors }\end{array}$ \\
\hline 14, Box 2 & $\begin{array}{l}\text { D17 cells stop responding to } \\
\text { dsRNA treatment or transient } \\
\text { transfection; D17 cultures } \\
\text { begin to die after more than } 20 \\
\text { passages }\end{array}$ & $\begin{array}{l}\text { Although D17 cells are an immortalized } \\
\text { line, they begin to lose their suscep tibility } \\
\text { to RNAi and reach a terminal growth } \\
\text { sometime after } 20 \text { passages }\end{array}$ & $\begin{array}{l}\text { Consider ordering new cells or thawing fresh } \\
\text { aliquots of D17 cells }\end{array}$ \\
\hline 15,17 & $\begin{array}{l}\text { Cells do not adhere and } \\
\text { migrate on ECM-cross- linked } \\
\text { glass surfaces }\end{array}$ & $\begin{array}{l}\text { This could be due to an inefficient cross- } \\
\text { linking process or inefficacious ECM }\end{array}$ & $\begin{array}{l}\text { If you are using a UV cross-linking treatment, } \\
\text { determine the power output of the UV source or } \\
\text { use a plasma treatment system. D17 migration can } \\
\text { be impaired by too-low or too-high concentrations } \\
\text { of ECM. The user should empirically test a range } \\
\text { of ECM concentrations to determine the optimal } \\
\text { concentration per ECM batch (see Experimental } \\
\text { design) }\end{array}$ \\
\hline
\end{tabular}

